\title{
Konzeptionalisierung und Messung formalen Institutionenwandels - Das Beispiel parlamentarische Regeln
}

\author{
Ulrich Sieberer · Peter Meißner · Julia F. Keh • Wolfgang C. Müller
}

Zusammenfassung Die Beschreibung und Erklärung von Institutionenwandel ist eine zentrale Herausforderung des neoinstitutionalistischen Forschungsprogramms. Dieser Aufsatz diskutiert zentrale konzeptionelle, methodische und forschungspraktische Probleme bei der Analyse des Wandels formaler Institutionen. Konzeptionell werden vier Analyseansätze diskutiert, die sich hinsichtlich des Trade-Offs zwischen Breite und analytischer Tiefe unterscheiden. Methodisch entwickeln wir Werkzeuge, durch die sich die Identifikation von Änderungen in formalen Regeln

Elektronisches zusätzliches Material: Die Online-Version dieses Artikels (doi: 10.1007/s12286014-0216-7) enthält zusätzliches Material, welches für autorisierte Benützer zugänglich ist.

Eine frühere Version dieses Artikels wurde auf der Jahrestagung 2013 der DVPW-Sektion Methoden in Konstanz vorgestellt. Wir danken den Teilnehmern der Tagung sowie zwei anonymen Gutachtern der ZfVP für wertvolle Hinweise. Unser besonderer Dank gilt unserem großen Team studentischer Hilfskräfte in Konstanz und Mannheim, ohne die die jahrelange Erhebung, Aufbereitung und Kodierung derart umfangreicher Primärdaten nicht möglich gewesen wäre. Die Forschung zu diesem Beitrag wurde oder wird gefördert von der Deutschen Forschungsgemeinschaft (Projekt SI 1470/2-1), dem Zukunftskolleg der Universität Konstanz und dem Mannheimer Zentrum für Europäische Sozialforschung (MZES) an der Universität Mannheim.

PD Dr. U. Sieberer $(\bowtie) \cdot$ P. Meißner, MA · J. F. Keh, MA

Fachbereich Politik- und Verwaltungswissenschaft und Zukunftskolleg, Universität Konstanz, 78457 Konstanz, Deutschland

E-Mail: ulrich.sieberer@uni-konstanz.de

P. Meißner, MA

E-Mail: peter.meissner@uni-konstanz.de

J. F. Keh, MA

E-Mail: julia.keh@uni-konstanz.de

Prof. Dr. W. C. Müller

Institut für Staatswissenschaft, Universität Wien,

Rooseveltplatz 3/1,

1010 Wien, Österreich

E-Mail: wolfgang.mueller@univie.ac.at 
weitgehend automatisieren und valide quantitative Indikatoren des Umfangs von Textänderungen erheben lassen. Wir demonstrieren die praktische Anwendung der Analyseansätze und Werkzeuge an Daten zum Wandel parlamentarischer Regeln in europäischen Demokratien und diskutieren weitere Anwendungsfelder.

Schlüsselwörter Automatisierter Textvergleich · Institutionenwandel · Messung · Parlamentarische Regeln

\title{
Conceptualizing and measuring change in formal institutions: The case of parliamentary rules
}

\begin{abstract}
Describing and explaining institutional change constitutes a major challenge for the new institutionalist research program. This article discusses key conceptual, methodological, and practical issues in analyzing change in formal institutions. Conceptually, it suggests four analytical approaches that differ with regard to the trade-off between breadth of coverage and analytical depth. Methodologically, we develop new software tools that allow a largely automated identification of changes in formal rules and that permit a valid, quantitative measurement of the amount of changes in a text. We demonstrate the application of the four approaches and the new tools using data on changes in parliamentary rules in European democracies and discuss further fields of application.
\end{abstract}

Keywords Automated text Comparison - Institutional change - Measurement · Parliamentary rules

\section{Einleitung}

Institutionen sind spätestens seit den 1990er Jahren wieder ins Zentrum politikwissenschaftlicher Analysen gerückt. Der Schwerpunkt lag dabei lange auf den Folgen von Institutionen für Verhalten und Outputs und basierte auf der Annahme, dass Institutionen als exogen und stabil angesehen werden können. Diese Annahme wird in jüngerer Zeit zunehmend in Frage gestellt, so dass Fragen von Institutionengenese, Institutionendesign und Institutionenwandel zu einer zentralen Forschungsfront des neoinstitutionalitischen Forschungsprogramms (sowohl in der rational choice als auch in der historischen Variante) geworden sind. Im Mittelpunkt stehen dabei zwei eng verknüpfte Fragen: Warum variieren institutionelle Regeln über Länder hinweg? Und in welchem Ausmaß und warum werden institutionelle Regeln über Zeit verändert? Diese Fragen werden sowohl theoretisch intensiv diskutiert (z. B. Ostrom 2005; Pierson 2004) als auch hinsichtlich zentraler Institutionen moderner Demokratien wie Wahlsysteme, territoriale Gewaltenteilung oder grundlegende Verfassungsordnungen empirisch untersucht (z. B. Benoit 2007; Harfst 2013; Renwick 2011; Benz und Behnke 2009; Kaiser 2002).

Die empirische Analyse institutionellen Wandels wirft indes eine Reihe von konzeptionellen und methodischen Fragen auf, die in der Literatur bislang kaum 
systematisch diskutiert wurden. Konzeptionell erfordert die Erklärung von Institutionenwandel zunächst die Unterscheidung verschiedener Typen institutioneller Reformen. Daneben ist zu klären, auf welcher Ebene und mit welcher Art von Indikatoren sich Institutionenwandel messen lässt sowie welche charakteristischen Stärken und Schwächen diese Analyseansätze jeweils aufweisen. Methodisch stellt sich zunächst die Frage, wie sich institutionelle Änderungen systematisch und effizient identifizieren lassen, insbesondere wenn eine Vielzahl an Regeln (beispielsweise der gesamte Verfassungsinhalt) über einen längeren Zeitraum und mehrere Länder hinweg analysiert werden soll. Darauf aufbauend müssen valide Maße für institutionelle Veränderungen entwickelt werden.

Dieser Aufsatz diskutiert diese Probleme und schlägt erste Schritte zu ihrer Lösung vor. Unsere Argumentation beschränkt sich auf formale Institutionen, d. $h$. rechtlich verbindliche und durchsetzbare Regeln, und blendet damit informellen Institutionenwandel, d. h. Veränderungen von Konventionen, Interpretationen und nicht kodifizierten Verhaltensregeln aus. Die Forschung zum Wandel politischer Institutionen teilt größtenteils diesen Fokus, auch wenn informelle Institutionen wichtige Folgen für politisches Verhalten haben können (z. B. Helmke und Levitsky 2004). Für die Konzentration auf formale Regeln sprechen vor allem deren Verbindlichkeit auch in Konfliktfällen sowie die Schwierigkeit, informelle Institutionen und ihre Veränderung reliabel zu messen. Zudem werden wichtige informelle Regeln häufig formalisiert, insbesondere wenn ihre Interpretation umstritten war. Daher sollten formale Institutionen zumindest in stark institutionalisierten Demokratien die wichtigsten und kontroversesten politischen Regeln beinhalten (Müller und Sieberer 2014; s. auch Marschall 2004).

Die in diesem Aufsatz entwickelten konzeptionellen Unterscheidungen, methodischen Werkzeuge und empirischen Indikatoren sind grundsätzlich auf alle formalen Institutionen anwendbar und ließen sich daher an verschiedensten Regeln wie Verfassungen, Wahlgesetzen oder Parteistatuten veranschaulichen. Wir verwenden zur Illustration Reformen parlamentarischer Geschäftsordnungen, die aus vier Gründen besonders geeignetes Anschauungsmaterial bieten. Erstens sind diese Regeln, im Gegensatz zu beispielsweise Verfassungen, meist sehr präzise formuliert, so dass sich Bedeutung und Folgen von Änderungen relativ gut prognostizieren lassen. Zweitens regulieren parlamentarische Geschäftsordnungen sehr direkt alltägliche politische Auseinandersetzungen, was politischen Akteuren große Anreize für eine strategische Veränderung dieser Regeln gibt. Drittens sind sie in den meisten Ländern leicht änderbar (Müller 2002), was wiederum empirisch zu häufigen und teils umfassenden Reformen führt. Dadurch entstehen viertens bei einer Analyse über Zeit große Datenmengen, was einerseits effiziente und weitgehend automatisierte Analysewerkzeuge besonders dringlich macht und andererseits ausreichend Material für die Illustration verschiedener Analyseansätze samt ihrer jeweiligen Stärken und Schwächen sowie der Komplementarität der Ansätze hervorbringt.

Unsere weitere Argumentation erfolgt in drei Schritten. In Abschnitt 2 besprechen wir zentrale konzeptionelle Unterscheidungen hinsichtlich verschiedener Typen von Institutionenwandel und stellen vier idealtypische Analyseansätze vor, die unterschiedliche Schwerpunkte bezüglich des inhärenten Forschungsdesign-Trade-Offs zwischen Breite und analytischer Tiefe setzen. In Abschnitt 3 stellen wir Verfahren 
und Werkzeuge vor, die den zeitaufwändigen Prozess der Identifikation von Änderungen in formalen Regeln weitgehend automatisieren und dadurch massiv vereinfachen. Wir präsentieren zunächst vollautomatische und teilautomatische Verfahren zur Verknüpfung zusammengehöriger Textelemente über verschiedene Versionen eines Textkorpus (z. B. einer Rechtsnorm) hinweg, die in der Programmiersprache R implementiert sind. Diese neuen Algorithmen können zum systematischen Vergleich jeder Art von Texten genutzt werden und sind damit weit über die Analyse formaler Institutionen hinaus anwendbar. Anschließend entwickeln wir ein neues quantitatives Maß für den Umfang institutioneller Änderungen auf Grundlage veränderter Wortzahlen. Wir zeigen, dass diese Verfahren zu massiven Effizienzgewinnen führen und gleichzeitig valide Maße hervorbringen. In Abschnitt 4 illustrieren wir die vier Analyseansätze und unsere methodischen Werkzeuge an Daten zu parlamentarischen Regeländerungen in Westeuropa seit 1945. Im Schlussabschnitt diskutieren wir die Anwendbarkeit unseres Ansatzes in anderen Kontexten.

\section{Konzeptioneller Rahmen: Ansätze zur Analyse von Institutionenwandel}

\subsection{Konzeptionelle Unterscheidungen}

Unter den Begriff Institutionenwandel fallen sehr vielfältige Phänomene. Daher sind konzeptionelle Unterscheidungen unerlässlich, mit deren Hilfe sich diese Vielfalt in intern homogene Untergruppen unterteilen lässt, die denselben kausalen Mechanismen unterliegen (,causal homogeneity“). Der Nutzen der hier diskutierten Differenzierungen wird bei der illustrativen Anwendung unserer vier Analyseansätze in Abschnitt 4 deutlich.

Erstens lassen sich drei formale Änderungstypen geschriebener Regeln unterscheiden, nämlich Hinzufügungen im Regelungstext, Löschungen und Modifikationen. Diese drei Typen decken zusammen sämtliche logisch möglichen Änderungen ab. Sie sind jeweils definiert auf Ebene der grundlegenden Analyseeinheit innerhalb des analysierten Textkorpus. Diese Analyseeinheit kann beispielsweise aus Kapiteln, Paragraphen oder Absätzen einer Rechtsnorm bestehen. Hinzufügungen sind demnach in einer Reform eingefügte Einheiten, die in der älteren Fassung des Texts keine Entsprechung haben; Löschungen sind Einheiten, die nur in der älteren Version vorkommen; und Modifikationen bezeichnen Einheiten, die mit unterschiedlichem Wortlaut in beiden Versionen zu finden sind. Die Differenzierung dieser Änderungstypen ist für eine valide Messung des Umfangs institutioneller Reformen erforderlich (s. Abschnitt 3.2).

Zweitens lassen sich institutionelle Reformen anhand ihrer Folgen für politische Prozesse und Ergebnisse klassifizieren, insbesondere hinsichtlich der Frage, wer von einer Reform profitiert. Zentral ist hierbei die von George Tsebelis (1990, Kap. 4) geprägte Unterscheidung von Pareto-effizienten und redistributiven Reformen. Pareto-effiziente Reformen nützen allen (oder bei etwas laxerer Verwendung des Konzepts nahezu allen) an der Reformentscheidung beteiligten Akteuren unabhängig von ihrer Position im politischen Wettbewerb (beispielsweise ihrer aktuellen und künftigen Regierungsbeteiligung). Solche Reformen entstehen oft als Reaktion auf Änderungen 
im externen Umfeld oder als koordinierter Versuch von Entscheidungsträgern, Kosten im Sinne einer Kartelllogik zu externalisieren. Beispiele umfassen die Stärkung parlamentarischer Instrumente zur Beeinflussung von EU-Entscheidungen oder Regelungen zur Parteien- und Wahlkampffinanzierung, die allen im Parlament vertretenen Parteien nutzen (wenn auch auf Kosten der Steuerzahler und möglicherweise im Parlament nicht vertretener Parteien). Redistributive Reformen hingegen verändern die Verteilung von institutioneller Macht, Geld oder anderen Ressourcen zwischen den am Reformprozess beteiligten Akteuren und stellen bestimmte Akteure auf Kosten anderer besser. Diesem Reformtyp sind beispielsweise Wahlsystemwechsel mit Einfluss auf die Chancen kleinerer Parteien, die Dezentralisierung von Entscheidungsbefugnissen oder die Stärkung bzw. Schwächung von Minderheitsrechten in Parlamenten zuzuordnen. Die Unterscheidung von effizienten und distributiven Reformen ist insbesondere für die Erklärung von Institutionenwandel wichtig, da diese Reformtypen aufgrund der unterschiedlichen Verteilung von Gewinnen verschiedenen Dynamiken folgen dürften (Sieberer und Müller 2014; Sieberer et al. 2011).

\subsection{Vier Analyseansätze}

Ein komplexes Phänomen wie Institutionenwandel lässt sich auf verschiedene Arten untersuchen. Wir unterscheiden vier idealtypische Analyseansätze für Reformen formaler Regeln. Diese Ansätze variieren in ihrer Position zu der jedem Forschungsdesign inhärenten Abwägung zwischen einer „Logik der Breite“ und einer „Logik der Tiefe" (Gschwend und Schimmelfennig 2007). Aufgrund ihrer jeweiligen Schwerpunkte eignen sich die vier Ansätze zur Beantwortung unterschiedlicher Fragen. In der Zusammenschau ergeben sie zudem ein umfassendes Bild des Wandels formaler Institutionen. Tabelle 1 fasst die Analyseansätze hinsichtlich ihrer Ziele, der verwendeten Datenbasis, möglichen Indikatoren und ihren spezifischen Stärken sowie den damit einhergehenden Grenzen zusammen.

Der erste Ansatz macht Aussagen über institutionelle Reformen auf der Makroebene. Diese Aussagen können entweder qualitativ als Einschätzung der Gesamtreform erfolgen (z. B. als ,umfassend“, ,,weitreichend“, oder „,marginal“) oder mit quantitativen Indikatoren gemessen werden, beispielsweise als Anzahl geänderter Worte gegenüber der vorherigen Fassung. Beispiele dieses Ansatzes sind die Kartierung von Wahlsystemreformen in westeuropäischen Demokratien (Katz 2005), Daten zur Änderungshäufigkeit von Verfassungen (Lutz 1994) sowie quantitative Beschreibungen der Veränderung parlamentarischer Geschäftsordnungen (Sieberer et al. 2011; s. u. Abschnitt 4.1). Die zentralen Ziele dieses Ansatzes sind die Beschreibung grundlegender Muster von institutioneller Stabilität und Veränderung sowie die Identifikation großer Reformen, die eine genauere Analyse rechtfertigen. Seine Stärke liegt im umfassenden Zugriff auf sämtliche Veränderungen institutioneller Regelungen, wodurch verzerrte Aussagen auf Grundlage einer nicht zufälligen Auswahl für Fallstudien verhindert werden. Der Ansatz ist besonders nützlich für die Analyse umfangreicher Regeln, die häufigen Änderungen unterliegen. Der Makroansatz stößt aber an zwei wichtige Grenzen. Erstens erlaubt er, insbesondere in seiner quantitativen Ausprägung, keine direkten Aussagen über die substanzielle Bedeutung von Reformen, da Änderungen sowohl zentrale als auch relativ unwichtige techni- 


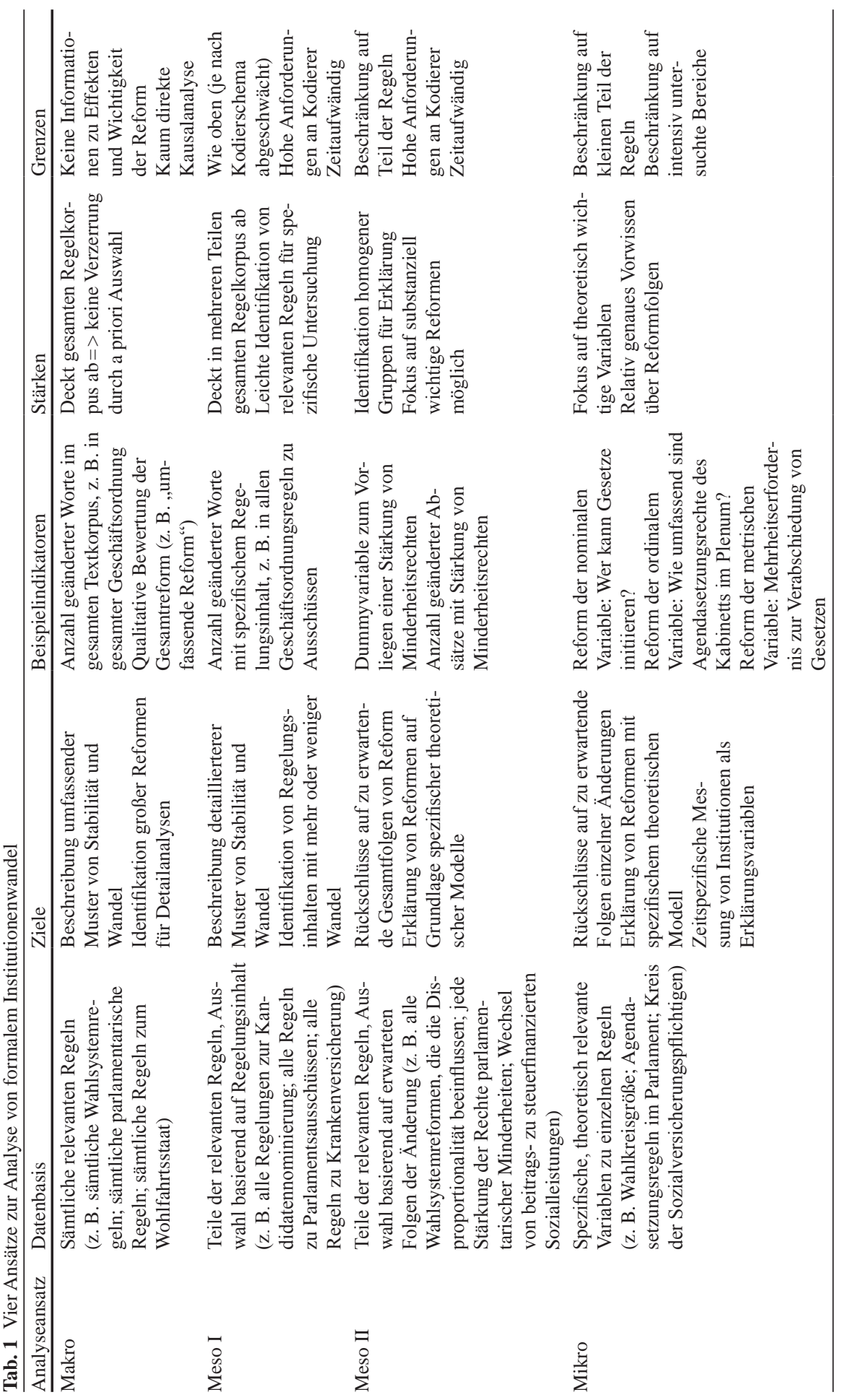


sche Regelungen betreffen können. Die substanzielle Wichtigkeit von Reformen lässt sich nur durch die Einbeziehung des Regelungsinhalts beurteilen (s. dazu den Meso II-Ansatz unten). Zweitens ignoriert er Unterschiede hinsichtlich der zu erwartenden Reformfolgen, differenziert beispielsweise nicht zwischen effizienten und redistributiven Reformen. Schließlich eignen sich Makrodaten nur dann als abhängige Variable zur Überprüfung von Erklärungsmodelle, wenn für sämtliche Reformen derselbe Kausalmechanismus angenommen werden kann, was beispielswiese bei parlamentarischen Regeländerungen, die effiziente und redistributive Reformen enthalten, nicht der Fall ist.

Der zweite und dritte Ansatz sind auf der Mesoebene angesiedelt und führen auf unterschiedliche Weisen Differenzierungen innerhalb der untersuchten Regelungen ein. Auch der als „Meso I“ bezeichnete Ansatz untersucht den gesamten relevanten Textkorpus, differenziert diesen aber nach dem Regelungsinhalt. Beispielsweise kann eine Studie zu Wahlsystemwandel Veränderungen in der Proportionalität der Mandatszuteilung von solchen hinsichtlich der Personalisierung des Wahlsystems trennen (Renwick 2011), eine Untersuchung zum Wandel des Wohlfahrtsstaats kann zwischen Subsystemen wie Krankenversicherung und Arbeitslosenversicherung differenzieren (Allan und Scruggs 2004), oder Analysen parlamentarischer Regeländerungen können zwischen Regeln zu Entscheidungen im Plenum, zur internen Organisation und zur Kontrolle der Regierung unterscheiden (Sieberer et al. 2011; s. u. Abschnitt 4.2). Auf diese Untergruppen können dann die oben beschriebenen Indikatoren zu Existenz und Umfang von Reformen angewendet werden. Der Meso I-Ansatz teilt die Stärken des Makroansatzes, erlaubt aber detailliertere Beschreibungen. Zudem ermöglicht eine Klassifizierung sämtlicher zugrundeliegenden Regeln anhand ihrer Inhalte die Identifikation von Bereichen, in denen Institutionenwandel mehr oder weniger häufig auftritt und kann als Grundlage für die gezielte Auswahl von Regeln bestimmten Inhalts dienen. Allerdings ist der Ansatz in der Kodierung ressourcenintensiv, insbesondere bei umfangreichen Textkorpora. Zudem treffen die Beschränkungen des Makroansatzes auch auf diesen Analyseansatz zu, wenn auch in geringerem Ausmaß, wenn ein sehr detailliertes inhaltliches Kodierschema verwendet wird.

Der dritte Analyseansatz - Meso II - klassifiziert Regeländerungen anhand ihrer erwarteten Folgen und konzentriert sich anschließend auf Reformen mit bestimmten Effekten, z. B. nur distributive Reformen wie Wahlsystemänderungen, die die Disproportionalität des Systems verändern (Harfst 2013), oder nur Parlamentsreformen, die Minderheitenrechte stärken oder schwächen (Binder 1996; Schickler 2000; s. u. Abschnitt 4.3). Der Ansatz dient vor allem dazu, Teilmengen von Reformen zu identifizieren, die denselben kausalen Dynamiken unterliegen und daher mit demselben theoretischen Modell erklärt werden können. Zudem kann im Rahmen der qualitativen Kodierung von Veränderungen prinzipiell auch deren substanzielle Wichtigkeit beurteilt werden, so dass eine begründete Fokussierung auf wichtige Reformen möglich wird. Im Vergleich zu den bisher diskutierten Ansätzen sind Daten nach dem Meso II-Ansatz allerdings mit größerer Unsicherheit behaftet, da die Wirkung institutioneller Reformen insbesondere bei Detailänderungen nur schwer abschätzbar ist. Entsprechend aufwändig und sensitiv ist die Kodierung. Zudem blendet eine fokussierte Analyse von Änderungen mit bestimmten Effekten zwangsläufig andere 
Reformen aus, so dass auf Grundlage dieses Ansatzes allein keine umfassenden Aussagen über institutionelle Stabilität und Wandel möglich sind.

Der vierte Ansatz analysiert Änderungen spezifischer institutioneller Variablen auf der Mikroebene. Der Fokus liegt auf der Veränderung von Variablen, deren Relevanz in früheren theoretischen und/oder empirischen Arbeiten herausgestellt wurde, wie beispielsweise die Wahlkreisgröße (Taagepera und Shugart 1989), parlamentarische Agendasetzungsregeln (Döring 1995; s. u. Abschnitt 4.4) oder die von einem wohlfahrtsstaatlichen Programm betroffenen Personengruppen (Korpi und Palme 1998). Dieser Ansatz erlaubt größere analytische Tiefe, da die Folgen institutioneller Reformen gut abschätzbar sind. Die Breite einer solchen Analyse ist hingegen notwendigerweise auf wenige Regeln beschränkt. Zudem läuft der Ansatz Gefahr, Regelungsbereiche zu vernachlässigen, die in der bisherigen Forschung wenig Beachtung gefunden haben. Das mag für intensiv erforschte Institutionen wie das Wahlsystem unproblematisch sein, führt aber zu Problemen bei Regeln, über deren theoretische und substanzielle Bedeutung aufgrund fehlender Forschung wenig systematisches Wissen existiert, wie beispielsweise parlamentarische Regeln zu Debattenformaten und zur Verteilung von Redezeiten (Müller und Sieberer 2014).

\section{Werkzeuge und Messinstrumente zur empirischen Erfassung formalen Institutionenwandels}

In diesem Abschnitt stellen wir zunächst Werkzeuge vor, mit deren Hilfe sich Änderungen in großen Textkorpora systematisch und effizient identifizieren lassen. Darauf aufbauend schlagen wir neue Messinstrumente zur quantitativen Erfassung des Umfangs institutioneller Änderungen auf der Makroebene vor. Die Logik der Werkzeuge und Indikatoren wird teils an fiktiven Beispielen, teils an realen Daten zu parlamentarischen Regeländerungen demonstriert. Eine ausführlichere Illustration ihrer empirischen Anwendung erfolgt in Abschnitt 4.

\subsection{Die Verknüpfung zusammengehöriger Texteinheiten}

Analysen formalen Institutionenwandels stehen zunächst vor der Herausforderung, Änderungen der relevanten Textbasis (beispielsweise der gesamten parlamentarischen Geschäftsordnung) systematisch zu identifizieren. ${ }^{1}$ Dieser Schritt ist bei der Analyse großer Textmengen sehr aufwändig und fehleranfällig. Daher stellen wir automatisierte Verfahren zur Verknüpfung zusammengehörender Texteinheiten über verschiedene Versionen von Dokumenten hinweg vor und diskutieren deren Nutzen für die oben unterschiedenen Analyseansätze.

\footnotetext{
${ }^{1}$ Unser Verfahren setzt voraus, dass die relevanten Regeln in einem klar abgrenzbaren und der Forschung zugänglichen Textkorpus enthalten sind. Diese Voraussetzung ist unproblematisch, wenn man Institutionen als formale Regeln konzeptualisiert, kann aber problematisch sein, wenn auch umfassende Policyregime wie der Wohlfahrtsstaat als Institution betrachtet werden, da sich für diese nur schwer ein geschlossener Textkorpus aller relevanten Regeln identifizieren lässt. Wir danken einem anonymen Gutachter für diesen Hinweis.
} 
Tab. 2 Ein fiktives Beispiel für den Vergleich von zwei Versionen eines Gesetzes

\begin{tabular}{|c|c|c|c|}
\hline \multicolumn{2}{|c|}{ Beispielgesetz Version 1} & \multicolumn{2}{|c|}{ Beispielgesetz Version 2} \\
\hline$\overline{P n r}$ & Text & Pnr & Text \\
\hline 1 & $\begin{array}{l}\S 1 \text { Dingse und Dängse sind hiermit } \\
\text { eingeführt }\end{array}$ & 1 & $\begin{array}{l}\S 1 \text { Dingse und Dängse sind hiermit } \\
\text { eingeführt }\end{array}$ \\
\hline 2 & $\begin{array}{l}\S 2 \text { Das Halten eines Dingses ist ab } \\
\text { einem Alter von } 25 \text { Jahren erlaubt }\end{array}$ & 2 & $\begin{array}{l}\S 2 \text { Das Halten eines Dängses ist } \\
\text { grundsätzlich ab einem Alter von } 5 \\
\text { Jahre erlaubt bedarf aber der Zustim- } \\
\text { mung der Eltern. Genaueres regelt das } \\
\text { Jugendschutzgesetz }\end{array}$ \\
\hline 3 & $\begin{array}{l}\S 3 \text { Ausnahmereglungen } \S 2 \text { betreffend } \\
\text { werden von Fall zu Fall vom zuständi- } \\
\text { gem Amt geregelt }\end{array}$ & 3 & $\begin{array}{l}\text { § } 3 \text { Das Halten eines Dingses ist ab } \\
\text { einem Alter von } 20 \text { Jahren erlaubt. } \\
\text { Früher nur wenn bereits ein Dängs für } \\
\text { mindestens } 2 \text { Jahre ohne Unterbre- } \\
\text { chung gehalten wurde }\end{array}$ \\
\hline 4 & $\begin{array}{l}\S 4 \text { Das Halten eines Dängses ist } \\
\text { grundsätzlich ab einem Alter von } 5 \\
\text { Jahre erlaubt bedarf aber der Zustim- } \\
\text { mung der Eltern. Genaueres regelt das } \\
\text { zuständige Amt auf Kreisebene }\end{array}$ & 4 & $\begin{array}{l}\text { § } 4 \text { Dingse und Dämgse gelten als } \\
\text { Sachen und sind bei Streitfragen als } \\
\text { solche zu behandeln }\end{array}$ \\
\hline 5 & $\begin{array}{l}\S 5 \text { Sowohl Dängse als auch Dingse } \\
\text { sind meldepflichtig }\end{array}$ & & \\
\hline
\end{tabular}

Veränderungen geschriebener Regeln lassen sich formal als eine Ansammlung von Hinzufügungen, Löschungen und Modifikationen beschreiben. Diese drei formalen Änderungstypen werden identifiziert, indem zwei Versionen eines Regelungstextes systematisch auf Ebene der relevanten Analyseeinheiten (Paragraphen, Absätze oder andere substanziell sinnvolle Einheiten) verglichen und zusammengehörige Einheiten einander zugeordnet werden. Diese Zuordnung - im Weiteren als „Verknüpfung“ bezeichnet - ist relevant für alle oben vorgestellten Analyseansätze: Das Verknüpfen ermöglicht es zunächst nachzuvollziehen, was mit jeder Untereinheit des Textkorpus in einer Reform geschieht. In einem zweiten Schritt können wir dann (1) den Gesamtumfang von Änderungen quantitativ messen (wichtig für den Makro-Ansatz), (2) inhaltliche Kodierungen zum Inhalt von Regeln für unveränderte Untereinheiten ohne erneute Kodierung übertragen (Meso I), (3) Veränderungen identifizieren, deren Effekte und substanzielle Wichtigkeit qualitativ kodiert werden können (Meso II) und (4) die Entwicklung einzelner Regelungen über Zeit verfolgen (Mikro).

Wir verdeutlichen den Vorgang des Verknüpfens an zwei Versionen eines fiktiven Gesetzes mit Paragraphen als Analyseeinheit (Tab. 2). Zunächst werden Analyseeinheiten identifiziert, die über beide Versionen hinweg identisch sind, in diesem Fall das Paragraphenpaar 1-1. Alle anderen Paragraphen unterliegen Veränderungen. Die Paragraphen 3 und 5 der älteren Version 1 wurden gelöscht, während Paragraph 4 der jüngeren Version 2 hinzugefügt wurde. Schließlich finden wir mit den Paragraphenpaaren 2-3 und 4-2 zwei Fälle, in denen Analyseeinheiten in beiden Versionen mit modifiziertem Wortlaut vorkommen. Diese Zuordnung wird in einen Datensatz überführt, der beide Versionen des Gesetzes enthält (Tab. 3). Zusammengehörige Paragraphenpaare werden in einer Zeile gespeichert, bei Löschungen und Hinzufügungen bleibt jeweils das Feld derjenigen Version leer, in welcher der Text nicht enthalten ist. 


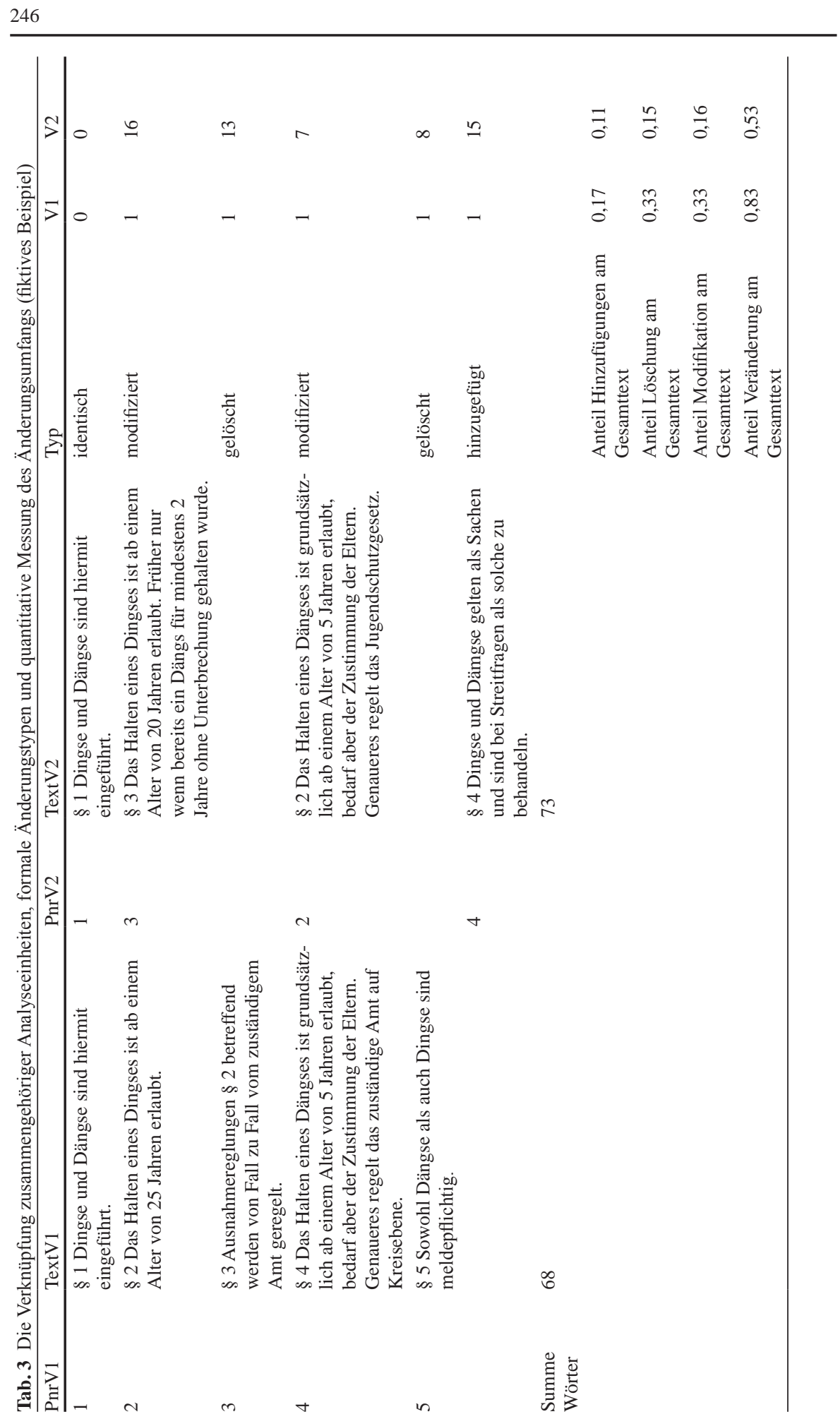


Die Aufgabe des Verknüpfens lässt sich grundsätzlich auf drei Arten umsetzen: Manuell, vollautomatisch und teilautomatisch. Ein manuelles Verknüpfen ist wie im vorigen Beispiel unter Verwendung herkömmlicher Tabellenkalkulationsprogramme möglich. Bei langen Texten und vielen Versionen ist dieses Verfahren allerdings extrem zeitaufwändig und fehleranfällig, insbesondere wenn Textteile im Rahmen gröBerer Umstrukturierungen durch den Text wandern und wenn Analyseeinheiten geteilt oder zusammengeführt werden. Daher sind stärker automatisierte Verfahren attraktiv.

Eine automatisierte Verknüpfung setzt ein quantitatives Maß der Textähnlichkeit voraus. Zur Messung der Ähnlichkeit oder Unähnlichkeit von Zeichenketten wurde eine Vielzahl von Distanzmaßen entwickelt. Van der Loo unterscheidet diese in „Editierdistanzen“, die die Anzahl von Operationen berechnen, mit denen eine Sequenz von Zeichen in eine andere überführt werden kann, „Q-gram-Distanzen“, welche die Häufigkeiten von Teilzeichenketten einer bestimmten Länge $q$ miteinander vergleichen, und „heuristische Distanzen“ als eine Residualkategorie komplexerer und wenig generalisierbarer Ansätze (siehe die Beispiele und Referenzen in van der Loo 2014). Distanzmaße kommen in verschiedenen wissenschaftlichen Disziplinen zum Einsatz, beispielsweise für Sequenzanalysen in Biologie, Sozialwissenschaften und Linguistik oder zur Verknüpfung von Untersuchungseinheiten (,record linkage“) über verschiedene statistische Datensätzen hinweg (Blanchard et al. 2014; Christen 2012; List 2014). Distanzmaße bilden zudem in der Informatik die Basis für Suchalgorithmen, Fehlerkorrekturen, Rechtschreibkontrollen und Versionskontrollsysteme. Letztere sind darauf ausgelegt, Texte (insbesondere Programmcode) zeilenweise zu vergleichen und dem Nutzer Einfügungen, Löschungen und Veränderungen von Textzeilen und Abschnitten anzuzeigen und sind damit für unsere Zwecke besonders relevant.

Unser Vorgehen bei der Verknüpfung von Textpassagen greift auf die Grundlogik von Versionskontrollprogrammen zurück, weist aber drei wichtige Unterschiede in der konkreten Ausführung auf. Erstens basiert unser Distanzmaß auf Worten anstatt auf Zeichen, da Worte natürliche sprachliche Sinneinheiten darstellen. Zweitens definieren wir die Ähnlichkeit zweier Textpassagen auf Grundlage der gemeinsam vorkommenden Worte, nicht aufgrund ihrer Abfolge. Nach unserer Logik sind zwei Textpassagen umso ähnlicher, je größer der Anteil gemeinsamer Worte ist, unabhängig davon, in welcher Reihenfolge diese Worte in den analysierten Texten vorkommen. Der Vergleich von Wortverteilungen erweist sich in der Praxis als erfolgreicher beim Auffinden inhaltlich zusammenhängender Textpassagen, weil Sätze in Rechtsnormen häufig umgestellt werden, ohne dass sich die dabei verwendeten Worte stark verändern. Die substanzielle Ähnlichkeit solcher Texte wird von Maßen wie der häufig verwendeten Levenshtein-Distanz (Levenshtein 1966), die auf der Abfolge von Zeichen beruhen, stark unterschätzt. Drittens implementieren wir den Prozess des Textvergleichs als neue Algorithmen in der Programmiersprache $\mathrm{R}$ anstatt auf fertige Textvergleichsprogramme wie Ultra Compare oder DiffDoc zurückzugreifen. Diese Entscheidung ermöglicht es uns, unsere Algorithmen genau auf die spezifischen Bedürfnisse unseres Projekts zuzuschneiden. Zudem kann so der gesamte Prozess der Datengenerierung, Datenanalyse und Ergebnispräsentation mit derselben 


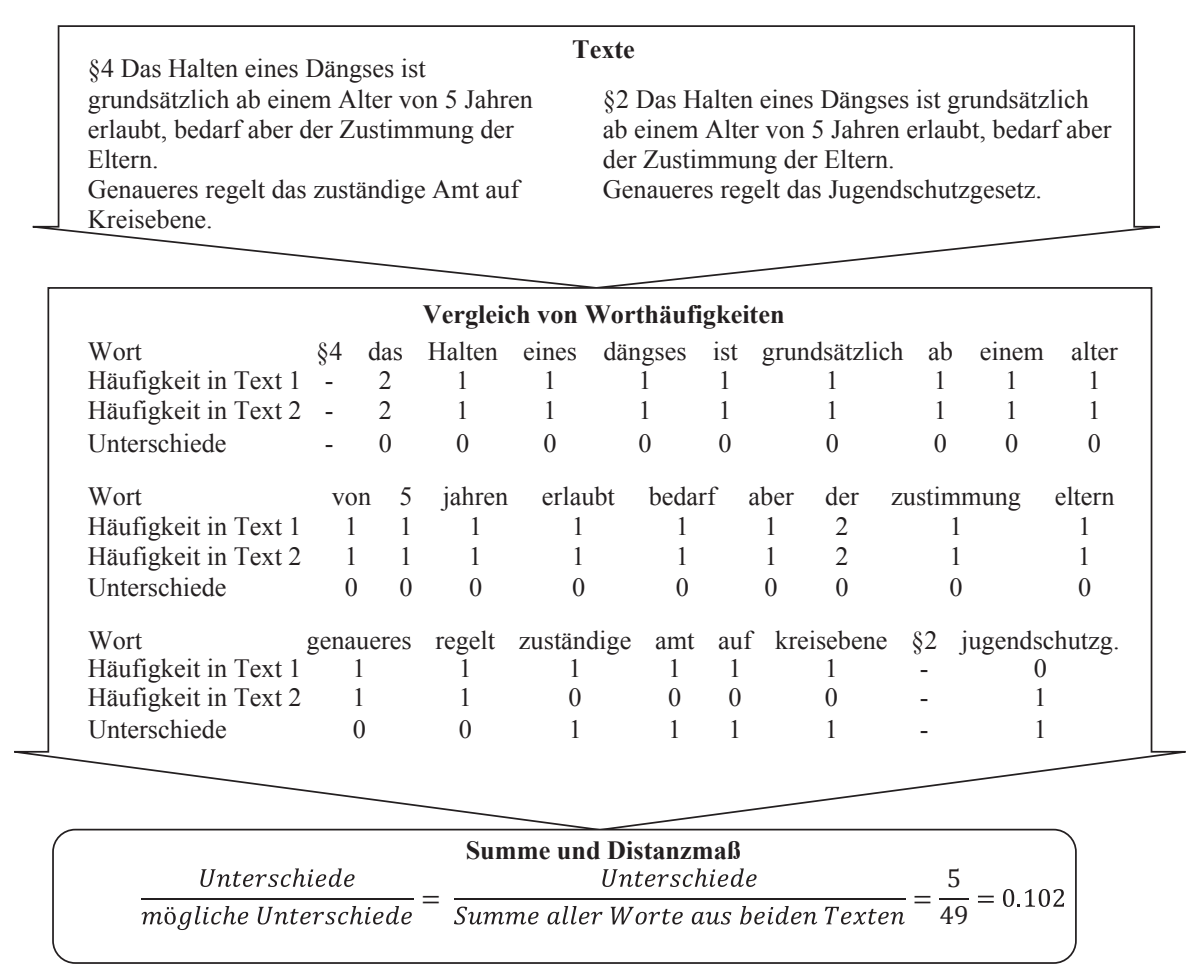

Abb. 1 Die Messung von Textähnlichkeit basierend auf Worthäufigkeiten (fiktives Beispiel)

Software durchgeführt werden, was die Fehleranfälligkeit reduziert und zu massiven Effizienzgewinnen führt. ${ }^{2}$

Zur Verknüpfung zusammengehöriger Texteinheiten vergleicht unser Algorithmus zunächst sämtliche möglichen Kombinationen von Analyseeinheiten zwischen zwei Textversionen und berechnet deren Ähnlichkeit basierend auf der relativen Summe der Abweichungen zwischen den Worthäufigkeiten in den beiden jeweils verglichenen Analyseeinheiten. Abbildung 1 demonstriert das Verfahren der Distanzmessung anhand des Paragraphenpaars 4-2 des Beispiels aus Tabelle 3. Das Programm vergleicht zunächst die Häufigkeit sämtlicher vorkommender Worte (außer der Paragraphennummerierung ${ }^{3}$ ) zwischen den beiden Versionen. In einem zweiten Schritt wird der Quotient aus der Anzahl der Wortunterschiede und der Summe aller Worte als Maß der Unähnlichkeit oder Distanz zwischen den beiden Analyseeinheiten für alle möglichen Kombinationen von Analyseeinheiten berechnet (im Fall des fiktiven

\footnotetext{
${ }^{2}$ Die Implementation des Algorithmus in R ist über GitHub verfügbar: https://github.com/petermeissner/ diffr. Durch den open source Charakter von R sind sämtliche Detailentscheidungen bei der Implementation unseres Algorithmus ersichtlich und können ggf. für andere Kontexte angepasst und verändert werden.

${ }^{3}$ Der Algorithmus ignoriert nur Paragraphennummerierungen am Beginn eines Absatzes, nicht hingegen Verweise auf andere Paragraphen innerhalb eines Absatzes.
} 
Gesetzes alle möglichen Kombinationen von jeweils einem Paragraph der älteren und jüngeren Version).

Auf Grundlage dieses Distanzmaßes kann die eigentliche Verknüpfung von Analyseeinheiten vollautomatisch oder teilautomatisch erfolgen. Beim vollautomatischen Verfahren bildet das Programm selbständig Paare von Analyseeinheiten nach abnehmender Ähnlichkeit. Einmal zugeordnete Analyseeinheiten werden aus der Menge möglicher Pendants herausgenommen. Anschließend wird das Paar mit der nächstgeringeren Ähnlichkeit gebildet, bis sämtliche Paare mit Wortüberschneidungen zugeordnet sind. Mit Hilfe dieses Verfahrens lassen sich große Textmengen relativ schnell verknüpfen. ${ }^{4}$ Ein Problem des vollautomatischen Vorgehens besteht allerdings darin, dass der Computer für nahezu jede Analyseeinheit ein scheinbar passendes Pendant findet, das mindestens ein identisches Wort enthält. Einige dieser Verknüpfungen erweisen sich bei näherer Analyse als falsch, d. h. die beiden Einheiten gehören inhaltlich nicht zusammen. Im Ergebnis werden Löschungen und Hinzufügungen systematisch unterschätzt und Modifikationen überschätzt.

Dieses Problems lässt sich mit unserem teilautomatischen Verknüpfungsverfahren lösen, das die Geschwindigkeit des vollautomatischen Verfahrens mit dem überlegenen inhaltlichen Verständnis menschlicher Kodierer verbindet. Bei diesem Verfahren werden nur identische Analyseeinheiten direkt vom Computer verknüpft. Für Einheiten ohne genaue Entsprechung schlägt der Computer auf Grundlage des Ähnlichkeitsmaßes mögliche Pendants vor, unter denen menschliche Kodierer die passende Verknüpfung auswählen oder die Analyseeinheit als nicht verknüpft (Hinzufügung oder Löschung) kodieren, wenn auch nach menschlichem Ermessen kein inhaltlich passendes Pendant existiert.

Die teilautomatische Verknüpfung ist zeitaufwändiger als die vollautomatische, gleichzeitig aber um ein Vielfaches effizienter als ein manuelles Vorgehen. Dies wird anhand einiger Zahlen deutlich: Alleine für die Analyse des Geschäftsordnungswandels in Belgien seit 1945 waren 59.467 Analyseeinheiten (Absätze) verteilt auf 45 Versionen zu bewältigen, von denen 33.209 tatsächlich verknüpft werden mussten (der Rest besteht aus inhaltsleeren Einheiten wie Überschriften und für unsere Zwecke irrelevanten Anhängen). Nachdem der Computer alle eindeutigen Verknüpfungen vorgenommen hatte, waren nur noch 5.925 Absätze (18\%) manuell zu bewältigen. Insgesamt erforderte die teilautomatische Verknüpfung etwa 30 Arbeitsstunden, eine vollständige manuelle Verknüpfung hätte rechnerisch etwa sechsmal länger gedauert. ${ }^{5}$

Der Nutzen der verschiedenen Verknüpfungsverfahren hängt stark vom Untersuchungsziel und dem Umfang der analysierten Textkorpora ab. Das vollautomatische Vorgehen ist nützlich, wenn man sich nur für globale Änderungsmaße interessiert ohne die Verknüpfungen weiter verwenden zu wollen und bereit ist, zugunsten größerer Geschwindigkeit eine gewisse Fehlermarge in Kauf zu nehmen (zu deren Größe s. u. Abschnitt 3.3). Auch die Übertragung inhaltlicher Kodierungen auf unveränderte Einheiten in späteren Versionen lässt sich mit den Resultaten des vollautomatischen

\footnotetext{
${ }^{4}$ Die benötigte Prozessorzeit für die vollautomatische Verknüpfung der in Abschnitt 4 vorgestellten 573 Versionen parlamentarischer Geschäftsordnungen liegt immer noch im Bereich von Tagen.

${ }^{5}$ Diese Schätzung ist sehr konservativ, da die Anzahl möglicher Kombinationen bei mehr Texteinheiten nicht linear ansteigt.
} 
Verfahrens problemlos realisieren. Für die übrigen Bearbeitungsschritte wie die Identifikation von Veränderungen zur qualitativen Kodierung oder das Verfolgen spezifischer Regeln über Zeit ist das vollautomatische Verknüpfen hingegen nicht geeignet, da es teilweise inhaltlich nicht korrespondierende Analyseeinheiten verknüpft. Das rein manuelle Verfahren empfiehlt sich höchstens für sehr kleine Textmengen und ist selbst dort fehleranfällig. Für die meisten Analysen formaler Regeländerungen auf Grundlage umfassender Textkorpora hingegen bietet sich unser teilautomatisches Verfahren mit seiner Verbindung von Geschwindigkeit und Genauigkeit an.

\subsection{Quantitative Messung des Änderungsumfangs auf Makroebene}

Mit Hilfe der oben vorgestellten Verknüpfungen lässt sich nun ein valider Indikator für den quantitativen Umfang von Textänderungen zwischen zwei Versionen entwickeln. Wir verdeutlichen unser Vorgehen an dem fiktiven Gesetz aus Tabelle 3. Die Gesamtveränderung des Texts setzt sich aus Hinzufügungen, Löschungen und Modifikationen zusammen. Ein simples Veränderungsmaß könnte schlicht die Häufigkeit dieser Änderungstypen auf Ebene der Analyseeinheit auszählen (Spalte V1 in Tab. 3). Allerdings ignoriert diese Messung den Änderungsumfang innerhalb von Analyseeinheiten, so dass die Veränderung eines Wortes mit einem vollständigen Umschreiben des gesamten Paragraphen gleichgesetzt wird. Um diese Unterschiede zu berücksichtigen, vergleichen wir mit einem zweiten in R implementierten Algorithmus den Inhalt der Analyseeinheiten auf Wortebene. Der Algorithmus erstellt für die beiden einander zugeordneten Analyseeinheiten je eine Worthäufigkeitstabelle und summiert die Unterschiede zwischen den beiden Versionen auf. Die Anzahl der nicht in beiden Versionen enthaltenen Worte ergibt einen validen Indikator des Veränderungsumfangs (Spalte V2).

Der untere Teil von Tabelle 3 verdeutlicht zwei Vorteile dieses Maßes. Erstens wird der Änderungsumfang in Bezug zur Gesamtlänge des Textes gesetzt. Es zeigt sich, dass Hinzufügungen und Löschungen gemessen an Wortzahlen deutlich weniger umfangreich sind als beim Blick auf den Anteil hinzugefügter und gelöschter Paragraphen. Zweitens erfasst das Maß bei Modifikationen nur tatsächlich geänderte Teile des Paragraphen und kann so punktuelle Änderungen von umfangreichen Umschreibungen unterscheiden. Schließlich demonstriert das Beispiel die Überlegenheit unseres Indikators gegenüber einem reinen Vergleich der Gesamtlängen der beiden Textversionen. Ein solcher Vergleich würde angesichts einer Differenz von nur fünf Worten von einer relativ kleinen Änderung ausgehen und damit weitreichende Umschreibungen innerhalb des Texts übersehen. Der Grund hierfür liegt darin, dass ein Textlängenvergleich nur Nettoveränderungen misst, nicht aber erfassen kann, dass Hinzufügungen und Löschungen sich gegenseitig aufheben und dass die Änderung eines Wortes sich nicht auf die Textlänge auswirkt. Dieses Phänomen wird in Abschnitt 4.1 empirisch illustriert.

Der hier entwickelte Indikator ist demnach ein überlegenes quantitatives Maß zum Umfang von Veränderungen innerhalb des analysierten Textkorpus. Allerdings stellt sich die Frage, ob er auch als Maß für den Umfang substanziell relevanten Institutionenwandels dienen kann. Dies setzt voraus, dass die substanzielle Wichtigkeit von Worten innerhalb des analysierten Texts in etwa gleich ist, so dass ein größerer Anteil 
geänderter Worte auch größere inhaltliche Veränderung impliziert. Diese Annahme ist nicht unproblematisch, auch wenn sie in ähnlicher Form in vielen quantitativen Textanalysetechniken getroffen wird (Huber und Shipan 2002; Slapin und Proksch 2014). Teils werden Texte umformuliert, ohne dass sich ihre substanzielle Bedeutung verändert, man denke beispielsweise an die Umschreibung der Geschäftsordnung des Belgischen Parlaments vom Passiv ins Aktiv im Jahr 1958. Hier kann großer Textwandel mit geringem inhaltlichem Wandel einhergehen. Umgekehrt können quantitativ minimale Veränderungen einzelner Worte massive substanzielle Reformen beinhalten, wenn z. B. ,einfache Mehrheit“" durch „Zweidrittelmehrheit“" ersetzt wird.

Letztlich lässt sich die genaue substanzielle Wichtigkeit institutioneller Reformen nur über eine Analyse des veränderten Inhalts erfassen - der quantitative Makroansatz allein kann also, wie in Abschnitt 2.2 konzeptionell diskutiert, Institutionenwandel nicht vollständig erfassen. Gleichzeitig zeigen die in Abschnitt 4 vorgestellten Daten, dass zumindest bei parlamentarischem Geschäftsordnungswandel die in der Literatur als qualitativ bedeutsam eingeschätzten Reformen meist auch quantitativ umfangreich waren. Insofern vermittelt der hier vorgeschlagene quantitative Indikator in den meisten Fällen auch ein qualitativ zutreffendes Bild der substanziellen Bedeutung von Reformen.

\subsection{Verzerrungen durch das vollautomatische Verknüpfungsverfahren und} Sprachunterschiede

Bei der Diskussion der vollautomatischen und teilautomatischen Verknüpfungsverfahren ist ein Trade-Off zwischen Schnelligkeit und Genauigkeit sichtbar geworden. Wie groß ist nun der Fehler des vollautomatischen Verfahrens und inwieweit kann dieser zugunsten von Geschwindigkeit in Kauf genommen werden, wenn man nur an groben quantitativen Maßen zur Veränderung des Textkorpus interessiert ist?

Wir können diese Frage durch einen Vergleich der „echten“, d. h. teilautomatisch erstellten Veränderungsmaße mit den im vollautomatischen Verfahren geschätzten Werten für aktuell 503 Geschäftsordnungen europäischer Parlamente beantworten, auf die wir beide Verfahren angewendet haben. Über alle Fälle hinweg beträgt die Korrelation zwischen diesen Werten 0,988, d. h. 97,5\% der Varianz in den ,echten" Werten kann durch die vollautomatische Schätzung rekonstruiert werden. Auf Ebene der einzelnen Länder entsteht ein ähnlich gutes Bild. Im schlechtesten Fall (Luxemburg) bilden die geschätzten Werte 89\% der tatsächlichen Varianz ab, in vielen Ländern ist die Korrespondenz mit Werten über $99 \%$ fast perfekt. Die Verzerrung durch das vollautomatische Verfahren lässt sich quantitativ mit einer einfachen Regression schätzen (Echter Wert $t_{i}=$ Bias $^{*}$ Geschätzter Wert $\left._{t}+\epsilon_{t}\right)$. Über alle Fälle hinweg ergibt sich ein Biasparameter von 1,11 , d. h. die echten Werte werden im Mittel leicht unterschätzt. Für die einzelnen Länder liegen die Werte zwischen 0,89 (Deutschland) und 1,27 (Italien). ${ }^{6}$ Diese Befunde zeigen, dass das vollautomatische Verfahren zumindest mit unseren Daten sehr gute, wenn auch nicht perfekte Maße für den Änderungsumfang liefert. Insofern bietet es die Möglichkeit, schnell einen

${ }^{6}$ Ein Streudiagramm der echten und geschätzten Werte sowie länderspezifische Korrespondenzmaße und Biasparameter finden sich in Abbildung A-1 und Tabelle A-1 im Online-Anhang. 
qualitativ zutreffenden Eindruck des Ausmaßes von Wandel in großen Textkorpora zu bekommen. ${ }^{7}$

Ein zweites Problem bei der quantitativen Messung von Veränderungen entsteht durch den Vergleich von Texten in verschiedenen Sprachen. Auf Wortzahlen basierende Veränderungsmaße müssen für die Neigung von Sprachen kontrollieren, mehr oder weniger Worte zum Ausdrücken desselben Inhalts zu verwenden (Huber und Shipan 2002). Dies kann mit Hilfe eines Sprachkorrekturfaktors geschehen, der die Textlänge in der jeweiligen Sprache ins Verhältnis zur Länge in einer Referenzsprache (in unserem Fall Englisch) setzt. Als Textgrundlage verwenden wir die in allen Amtssprachen der Europäischen Union verfügbare Charta der Grundrechte der Europäischen Union. Die oben entwickelten Veränderungsmaße werden standardisiert, indem man sie durch diese Korrekturfaktoren dividiert. ${ }^{8}$

\subsection{Weitere Nutzung der Verknüpfungen}

Die vorigen Abschnitte haben gezeigt, dass das Verknüpfen zusammengehöriger Texteinheiten über Versionen hinweg zentrale Bedeutung für die Identifikation institutioneller Änderungen sowie die quantitative Messung von deren Umfang hat. Darüber hinaus bieten die Verknüpfungen weitere Vorteile für die effiziente Kodierung und Analyse institutioneller Regeln und ihrer Veränderung. Erstens erlauben sie das automatische Übertragen von Kodierungen zum Regelungsinhalt. Dies ist besonders für den Analyseansatz Meso I bedeutsam, in dem der gesamte Textkorpus nach inhaltlichen Kriterien kodiert wird. Bei unveränderten Analyseeinheiten kann die bestehende Kodierung automatisch übertragen werden. Bei der Analyse parlamentarischer Geschäftsordnungen erweist sich diese Strategie als sehr erfolgreich, da im Regelfall große Teile des Textes unverändert bleiben. Im Schnitt wird die Kodierung eines Absatzes je nach Land zwischen acht und sechzehn Mal weitergegeben, so dass durch die automatische Übertragung rechnerisch zwischen $87 \%$ und $93 \%$ des ansonsten erforderlichen Kodieraufwands eingespart und zudem inkonsistente Kodierungen vermieden werden.

Zweitens erleichtert die Verknüpfung von Textpassagen die Kodierung spezifischer Änderungen in den Ansätzen Meso II und Mikro. Zum einen können unveränderte Passagen automatisch aus den zur Kodierung verwendeten Datensätzen herausgefiltert werden. Zum anderen unterscheidet sich der Kodierprozess zwischen Modifikationen einerseits und Hinzufügungen und Löschungen andererseits. Modifikationen können nur durch einen expliziten Vergleich zwischen der älteren und jüngeren Textversion kodiert werden. Die beiden anderen Änderungstypen hingegen lassen sich entweder aus sich selbst heraus verstehen oder entziehen sich einer Kodierung vollständig, weil ein aussagekräftiger Vergleichsmaßstab fehlt, an dem beispielsweise gemessen werden könnte, wie die Neuregelung institutionelle Macht zwischen Akteuren verschiebt.

\footnotetext{
${ }^{7}$ Ohne weitere Anwendungen unseres Verfahrens auf andere Textkorpora bleibt die Frage offen, wie generalisierbar die von uns gefundene hohe Übereinstimmung der Ergebnisse der beiden Verfahren ist.

${ }^{8}$ Die Werte für die verwendeten Sprachen sind in Tabelle A-1 im Online-Anhang aufgeführt.
} 


\section{Ein Beispiel aus der Forschungspraxis: Die Analyse parlamentarischer Regeländerungen in Westeuropa}

In diesem Abschnitt illustrieren wir die oben beschriebenen Analyseansätze und Werkzeuge anhand von Daten zu Reformen parlamentarischer Geschäftsordnungen in Westeuropa. Angesichts des konzeptionell-methodischen Fokus des Aufsatzes dient dieser Abschnitt alleine der Illustration der Analyseansätze und Werkzeuge, weshalb wir auf eine theoretische Diskussion der Ursachen parlamentarischer Regeländerungen und eine substanzielle Interpretation der empirischen Befunde verzichten. Auch die Auswahl der analysierten Länder orientiert sich am Ziel der Illustration (für erste theoretische und substanzielle Ergebnisse unserer Analysen siehe Sieberer und Müller 2014; Sieberer et al. 2011, 2014a, b).

\subsection{Der Makro-Ansatz: Quantitative Maße zur Veränderung parlamentarischer Regeln}

Auf der Makroebene lassen sich drei quantitative Indikatoren von Regeländerungen unterscheiden, die das Phänomen zunehmend genauer erfassen aber auch steigenden Erhebungsaufwand beinhalten: Die Reformanzahl, die Veränderung der Textlänge über Zeit sowie die Anzahl der pro Reform veränderten Worte. Diese Maße beziehen sich jeweils auf den gesamten Regelungsinhalt. Zur Illustration betrachten wir die Entwicklung des vollständigen Textkorpus parlamentarischer Geschäftsordnungen in 14 westeuropäischen Demokratien im Zeitraum von 1945 bzw. dem Beginn des aktuellen demokratischen Regimes bis Anfang 2010. ${ }^{9}$

Insgesamt haben in diesem Zeitraum 580 Veränderungen stattgefunden. Die meisten Reformen verzeichnet Schweden mit 110 Änderungen, die wenigsten Spanien mit lediglich zehn Fällen. Gemessen am Untersuchungszeitraum sind Änderungen in Schweden am häufigsten (1,72 Änderungen pro Jahr) und in Österreich am seltensten (0,2 Änderungen pro Jahr).

Die reine Reformanzahl ignoriert allerdings den Umfang von Reformen. Die Entwicklung der Textlänge gibt erste Aufschlüsse über den Reformumfang und die Entwicklung des formalen Regulierungsgrads parlamentarischer Prozesse. Abbildung 2 stellt die Textlängenentwicklung der Geschäftsordnungen in den 14 untersuchten Ländern in Englisch-äquivalenten Worten dar. Sie zeigt, dass der Umfang parlamentarischer Geschäftsordnungen deutlich angestiegen ist und dass sich das Ausmaß des Anstiegs zwischen Ländern unterscheidet. Bemerkenswert ist weiterhin, dass der Längenzuwachs in Österreich (dem Land mit den wenigsten Reformen) deutlich über dem in Schweden (dem Land mit den meisten Reformen) liegt. Dieser Befund unterstreicht, dass Reformhäufigkeit und -umfang nicht notwendig zusammenhängen und die reine Reformanzahl damit ein sehr verzerrtes Bild von Reformaktivität liefern kann.

Aber auch die Veränderung der Textlänge ist ein problematisches Maß, da sie den Gesamtumfang von Änderungen systematisch unterschätzt. Abbildung 3 verdeut-

${ }^{9}$ Die analysierten Länder sind Belgien, Dänemark, Deutschland, Frankreich, Island, Italien, Luxembourg, die Niederlande, Norwegen, Österreich, Portugal, Schweden, die Schweiz und Spanien. 

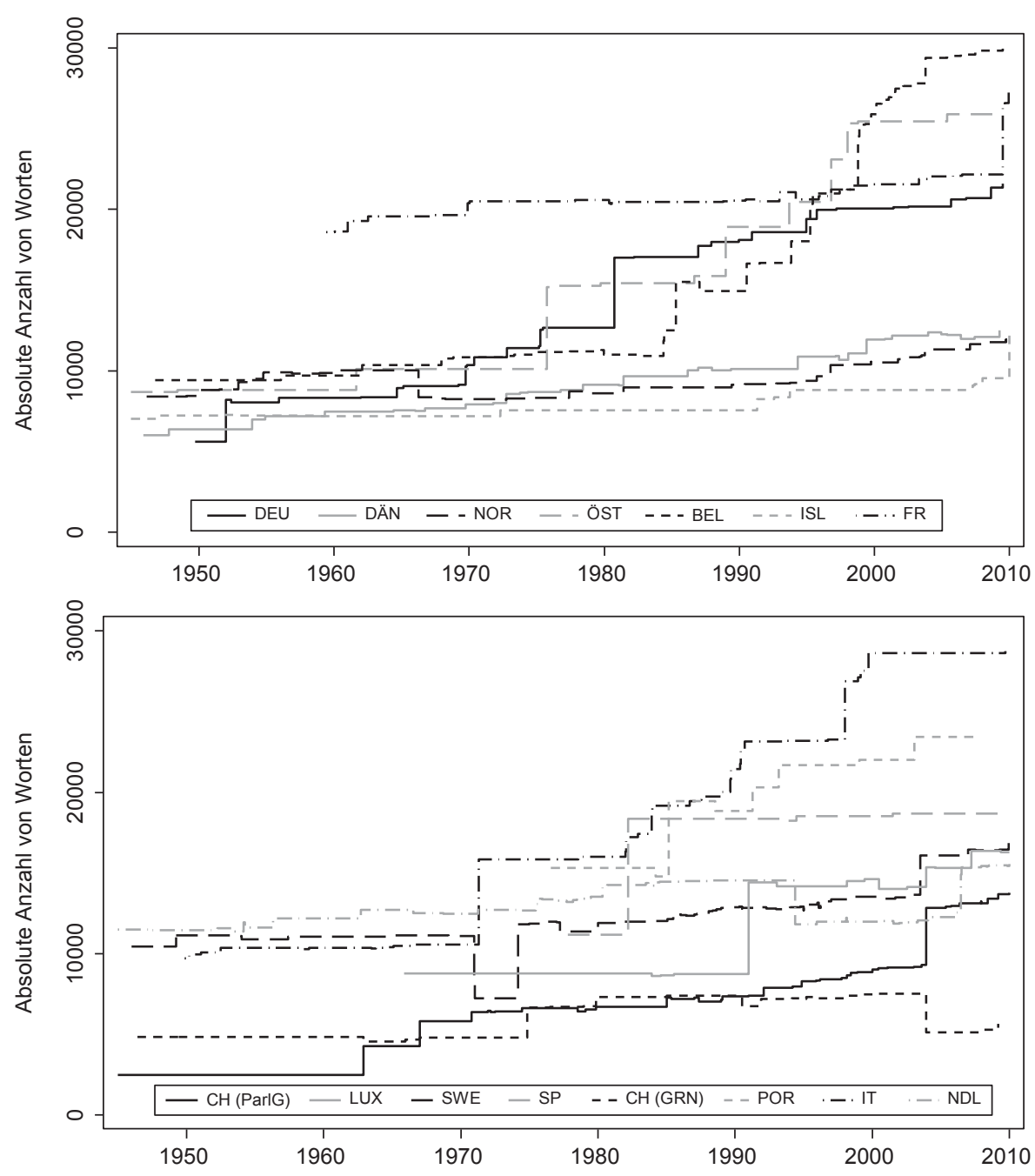

Daten für Spanien und Portugal seit der Demokratisierung.

Daten für Frankreich seit Beginn der 5. Republik.

In Luxemburg findet die erste Reform nach 1945 erst 1965 statt.

In der Schweiz sind Geschäftordnungsregeln auf das Parlamentsgesetz (ParlG) und das Geschäftsreglement des Nationalrats (GRN) verteilt.

Abb. 2 Veränderungen der Textlänge parlamentarischer Geschäftsordnungen in 14 Westeuropäischen Demokratien (in Englisch-Äquivalenten). (Quelle: Eigene Daten)

licht dies exemplarisch an der Anzahl der in jeder Reform in Österreich geänderten Worte für die drei formalen Änderungstypen sowie die Veränderung der gesamten Textlänge. Der Anstieg der Textlänge (dargestellt durch die schwarze Linie mit Kreuz) liegt für die meisten Reformen deutlich unter der Summe von hinzugefügten, gelöschten und modifizierten Worten. Die Veränderung der Textlänge und die Summe der drei Änderungstypen (berechnet auf Grundlage der teilautomatischen Verknüpfungen) weisen in unserem Datensatz nur eine Kovarianz von $30 \%(r=0,55)$ 


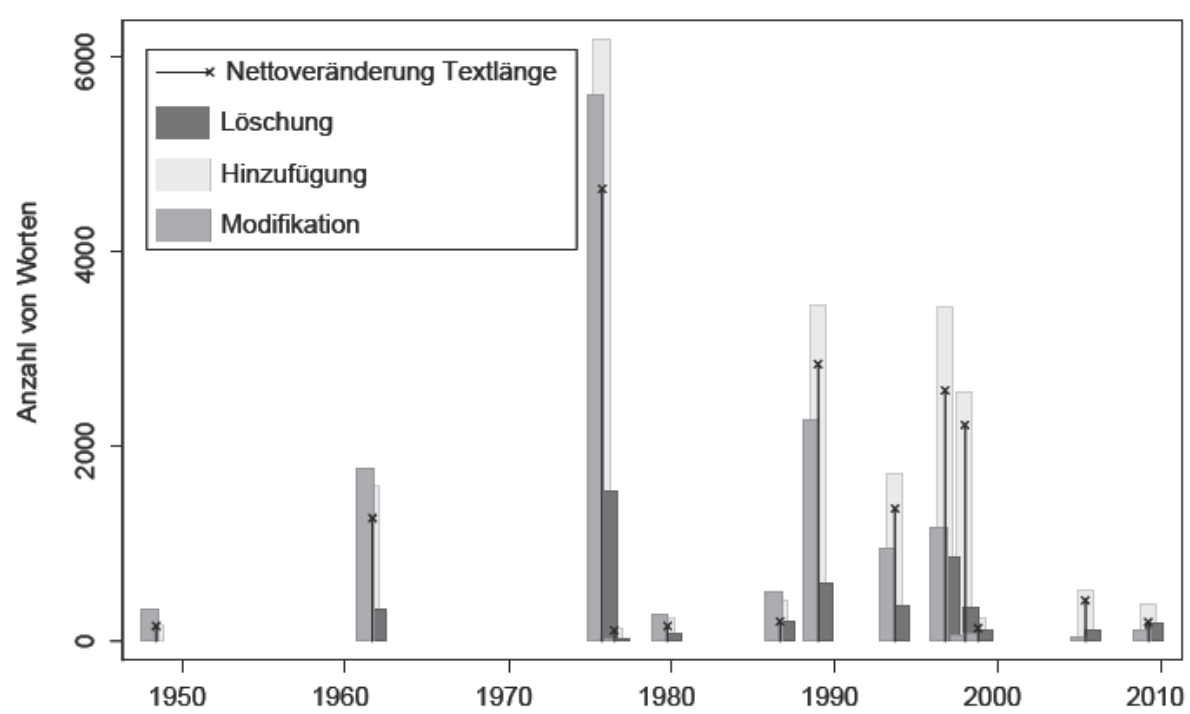

Abb. 3 Umfang von Geschäftsordnungsänderungen nach formalem Änderungstyp im Österreichischen Nationalrat (in Englisch-Äquivalenten). (Quelle: Eigene Daten)

auf. ${ }^{10}$ Dieser für eine Messung niedrige Wert zeigt, dass die Veränderung der Textlänge ein sehr unvollständiges $\mathrm{Maß}$ des tatsächlichen Änderungsumfangs ist - sie misst nur den Nettowandel, während die Bruttoveränderung deutlich höher liegt und häufig anders verteilt ist.

\subsection{Der Meso I-Ansatz: Die Veränderung parlamentarischer Regeln nach Tätigkeitsfeldern}

Der Meso I-Ansatz wendet die im letzten Abschnitt diskutierten quantitativen Maße einzeln auf inhaltlich definierte Teilbereiche eines Regelungskorpus an und identifiziert so Bereiche mit hoher oder niedriger Reformintensität. Wir illustrieren dies anhand der Veränderung der Textlänge von Geschäftsordnungsregeln zu fünf parlamentarischen Tätigkeitsfeldern: Entscheidungsfindung im Plenum, interne Organisation des Parlaments, Beziehung zu externen Organen und Kontrolle, Bestimmungen zum Ändern der Geschäftsordnung und eine Residualkategorie Sonstiges. Zur Generierung dieser Daten wurden sämtliche Geschäftsordnungsregeln auf Absatzebene einer von 80 exklusiven inhaltlichen Kategorien zugeordnet, die anschließend zu den fünf Tätigkeitsfeldern aggregiert wurden (s. ausführlich Sieberer et al. 2011).

Die exemplarische Analyse der Geschäftsordnung des Deutschen Bundestages in Abbildung 4 zeigt, dass das auf Makroebene beobachtete Längenwachstum auf Veränderungen in nahezu allen parlamentarischen Tätigkeitsfeldern inklusive der politisch besonders relevanten zurückgeht. Stärker qualitative Analysen zeigen zudem, dass auch theoretisch und politisch zentrale Regeln verändert werden (s. u. Abschnitt 4.4). Eine interessante Ausnahme bildet die fast vollständige Stabilität von Regeln zur

\footnotetext{
${ }^{10}$ Diese Zahlen beruhen auf 503 Beobachtungen aus 12 Ländern.
} 


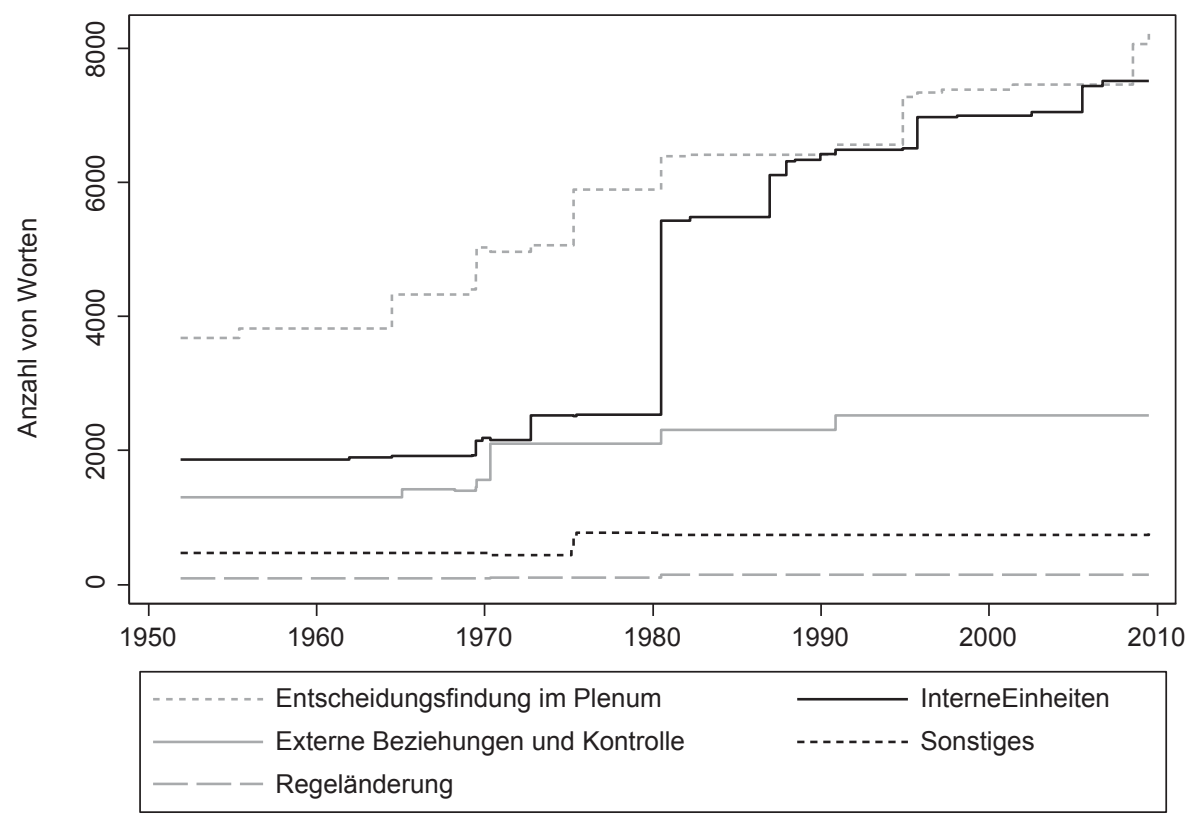

Abb. 4 Veränderungen der Geschäftsordnung des Deutschen Bundestages nach Gegenstandsbereichen (in deutschen Worten)

Änderung der Geschäftsordnung, die darauf hindeutet, dass diese als „Institutionen zweiter Ordnung“ exogen sind und daher institutionelle Reformprozesse tatsächlich strukturieren können (Diermeier und Krehbiel 2003).

Diese Befunde demonstrieren den Zusatznutzen aus der Verbindung quantitativer Indikatoren mit einer qualitativen inhaltsanalytischen Kodierung im Meso I-Ansatz. So lässt sich beispielsweise der Verdacht widerlegen, das massive Längenwachstum der Geschäftsordnung des Bundestages sei allein auf politisch relativ unwichtige Ergänzungen wie die Geheimschutzordnung zurückzuführen. Darüber hinaus erleichtert die inhaltliche Kodierung des gesamten Textkorpus Detailanalysen im Sinne des Mikro-Ansatzes, indem sie das Auffinden von Regeln zu bestimmten inhaltlichen Materien (beispielsweise zum Ausschusssystem, zu Fragerechten oder zur Rolle des Parlamentspräsidenten) massiv vereinfacht.

4.3 Der Meso II-Ansatz: Institutionelle Machtverschiebungen zwischen parlamentarischer Mehrheit und Minderheit

Der Meso II-Ansatz differenziert zwischen verschiedenen Reformen anhand ihrer erwarteten Effekte und identifiziert so kausal homogene Gruppen, an denen theoretische Erklärungsmodelle für Institutionenreformen adäquat überprüft werden können. Wir illustrieren diesen Ansatz an redistributiven Reformen, welche die institutionelle Machtverteilung zwischen parlamentarischer Mehrheit und Minderheit verändern. Hierzu wurden zunächst sämtliche Änderungen auf Absatzebene je nach ihren Auswirkungen auf diese Machtverteilung als mehrheitsstärkend, minderheitsstärkend 
oder neutral kodiert. In einem zweiten Schritt wurde auf dieser Grundlage der Charakter der gesamten Reform bestimmt.

Abbildung 5 zeigt exemplarisch für Deutschland, Frankreich, Österreich und Spanien die Anzahl von Absätzen, die pro Reform zugunsten der Mehrheit (in der Grafik die Linien nach oben) bzw. der Minderheit (nach unten) verändert wurden. Im Untersuchungszeitraum sind in Deutschland 15, in Frankreich 14, in Österreich zehn und in Spanien drei derartige Reformen zu beobachten. Der Reformumfang variiert beträchtlich. Während viele Reformen nur wenige Absätze zugunsten der Mehrheit oder Minderheit ändern, wird die Machtverteilung in einigen Fällen umfassend verändert. Zudem kombinieren viele Reformen Änderungen in beide Richtungen, was als Indiz für Kompromisse und Paketlösungen interpretiert werden kann. Dennoch lässt sich in den meisten Fällen eine dominante Reformrichtung identifizieren (in Abbildung 5 durch dicke Balken gekennzeichnet). Die mit unserem quantitativen Indikator erfasste Reformrichtung sowie die als besonders umfassend identifizierten Reformen stimmen mit der Einschätzung der qualitativen Literatur zu Parlamentsreformen in diesen Ländern überein, was die Validität des Maßes unterstreicht (zu Deutschland z. B. Marschall 1999; zu Österreich Fischer 1975, 2002; zu Frankreich Brouard 2011; zu Spanien Mellado Prado 1983). Diese Daten können als abhängige Variable zur Überprüfung eines theoretischen Erklärungsmodells für redistributiven Institutionenwandel in Parlamenten dienen (Sieberer und Müller 2014; Sieberer et al. 2014b).
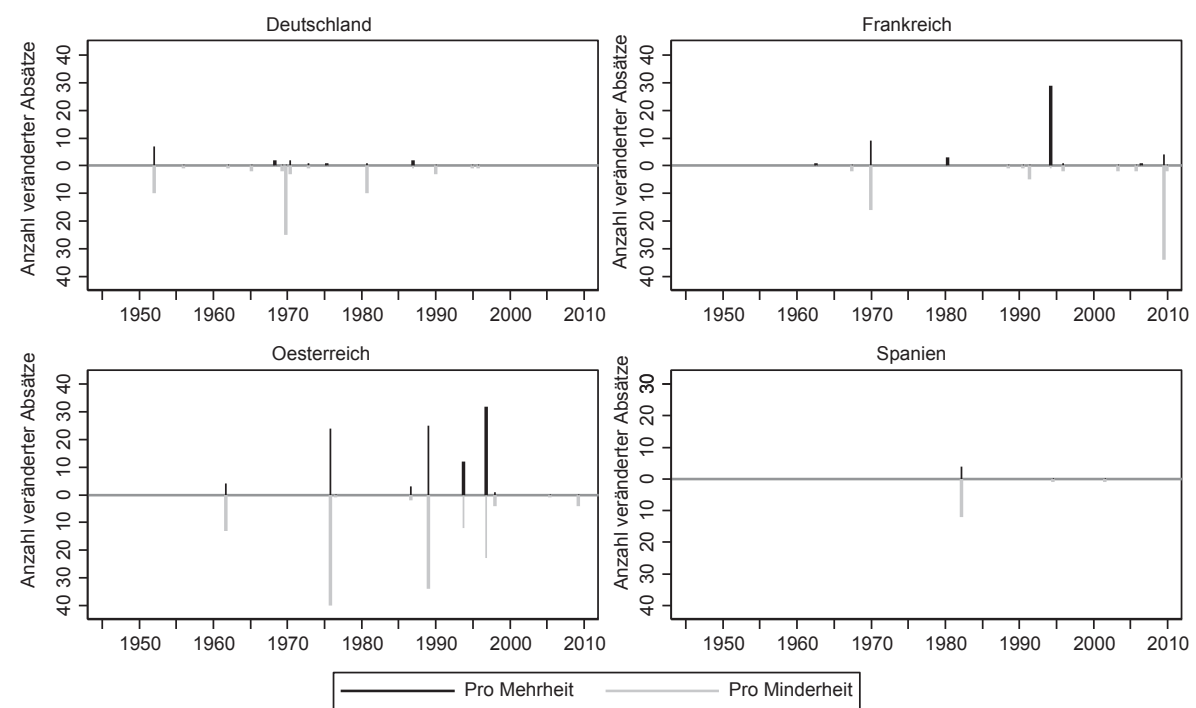

Die Kodierung der Gesamtreform ist durch dicke Balken gekennzeichnet.

Abb. 5 Veränderungen der institutionellen Machtverteilung zwischen parlamentarischer Mehrheit und Minderheit in vier westeuropäischen Demokratien 
4.4 Der Mikro-Ansatz: Die Veränderung von Agendasetzungsregeln über Zeit

Der Mikro-Ansatz analysiert Veränderungen einzelner, als wichtig erachteter Regeln über Zeit. Analysen derartiger Reformen sind einerseits per se interessant angesichts der Relevanz der analysierten Regeln für politisches Verhalten und Ergebnisse. Andererseits liefern sie zeitspezifische Maße institutioneller Variablen und ermöglichen damit eine valide Schätzung institutioneller Effekte in Zeitreihenanalysen. Im Kontext parlamentarischer Regeln ist gerade der zweite Punkt nicht zu vernachlässigen, da Studien parlamentarischen Verhaltens über Zeit fast ausnahmslos von der Annahme exogener und stabiler Regeln ausgehen - eine Annahme, die durch die oben demonstrierte Reformintensität zumindest in Frage gestellt wird.

Durch die Verknüpfung zusammengehöriger Textpassagen über alle Versionen einer Rechtsnorm hinweg sowie die inhaltliche Kodierung des Gesamtkorpus ist die Messung spezifischer Variablen über Zeit mit relativ geringem Aufwand möglich. Zunächst müssen die für eine Variable relevanten Textabschnitte identifiziert werden, wobei die Suche im Fall einer inhaltlichen Kodierung des Textkorpus nur einen kleinen Teil des Gesamttexts umfasst. ${ }^{11}$ Nach der Identifikation der relevanten Passagen zeigt das computergenerierte Ähnlichkeitsmaß direkt an, ob und wenn ja wann es zu Änderungen kam. Entsprechend müssen nur wenige Textversionen tatsächlich kodiert werden, während die Kodierung bei unverändertem Wortlaut mit den oben vorgestellten Werkzeugen weitergegeben werden kann.

Wir illustrieren den Mikroansatz an Veränderungen von zwei Agendasetzungsregeln im Deutschen Bundestag: Dem Zugang zur Tagesordnung im Plenum und der Beendigung von Debatten (zur deren theoretischer Relevanz s. Döring 1995; Sieberer 2006). Der Zugang zur Plenaragenda verläuft grundsätzlich über eine konsensuelle Entscheidung im Ältestenrat oder im Konfliktfall per Mehrheitsentscheidung im Plenum. Seit 1949 wurden Minderheitenrechte in diesem Prozess deutlich ausgebaut: Seit 1980 konnte der Antragsteller die Behandlung seiner Vorlage sechs Wochen nach ihrer Verteilung verlangen. Diese Frist wurde 1995 auf drei Wochen reduziert, so dass auch Minderheitsakteure heute garantierten und zeitnahen Zugang zur parlamentarischen Tagesordnung haben. Da das Herauszögern von Abstimmungen durch Endlosdebatten ein probates Obstruktionsmittel für parlamentarische Minderheiten sein kann, kennen fast alle modernen Parlamente Regeln zur Beendigung von Debatten (Cox und McCubbins 2011). Auch Bundestagsdebatten können grundsätzlich durch den Beschluss einer einfachen Mehrheit beendet werden. Zwischen 1949 und 1980 bestand die einzige Bedingung darin, dass nach dem Antragsteller oder Berichterstatter ein weiterer Abgeordneter zu Wort gekommen war. Seit 1980 hingegen muss jede Fraktion mindestens einmal gesprochen haben. Zudem wurde eine entsprechende Regel auch für Ausschussdebatten eingeführt. Auch bei der Beendigung der Debatte ist also eine Stärkung der Position von Minderheiten zu verzeichnen.

\footnotetext{
${ }^{11} \mathrm{Um}$ sämtliche relevanten Textstellen zu identifizieren empfiehlt es sich, die älteste und die jüngste sowie ggf. Versionen mit umfangreichen Änderungen zu analysieren, v. a. wenn es möglich erscheint, dass die zugrundeliegende Materie nur während eines Teils des Untersuchungszeitraums reguliert war.
} 


\section{Zusammenfassung und Ausblick}

Dieser Aufsatz hat zentrale konzeptionelle und methodische Herausforderungen bei der Analyse formalen Institutionenwandels diskutiert und Wege zu ihrer Lösung vorgeschlagen. Konzeptionell wurden vier idealtypische Ansätze zur Analyse institutioneller Reformen unterschieden, die in der Zusammenschau ein umfassendes Verständnis des Wandels formaler Institutionen erlauben. Zur Messung institutioneller Veränderungen wurden neue Verfahren vorgestellt, mit deren Hilfe zusammengehörige Textelemente in großen Textkorpora computergestützt identifiziert werden können. Diese Verknüpfungen erlauben eine effiziente Identifikation sämtlicher Regeländerungen zwischen verschiedenen Versionen von Texten sowie eine valide Messung des Umfangs von Textänderungen und führen zudem zu massiven Effizienzgewinnen bei der anschließenden Kodierung dieser Reformen. Durch diese Werkzeuge lassen sich umfassende Studien institutioneller Veränderungen über lange Zeiträume und eine Vielzahl von Ländern realisieren, die bislang schon durch den immensen Zeitaufwand beim Auffinden und Kodieren von Änderungen weitgehend verhindert wurden.

Die Anwendung der verschiedenen Analyseansätze sowie unserer neuen Analysewerkzeuge und Maße wurde am Beispiel parlamentarischer Geschäftsordnungsänderungen in europäischen Parlamenten illustriert. Angesichts des exemplarischen Charakters dieser Anwendung stellt sich die Frage, welche generelleren Schlüsse sich daraus über den Nutzen der verschiedenen Ansätze und Werkzeuge für die Analyse von Reformen in anderen institutionellen Kontexten ziehen lassen. Zunächst ist zu betonen, dass die vier Analyseansätze Makro, Meso I, Meso II und Mikro wie in Abschnitt 2.2 dargestellt unterschiedliche Stärken und Schwächen haben und daher als komplementär, nicht als Substitute anzusehen sind.

Wenn der untersuchte Regelungskorpus, wie im Fall parlamentarischer Regeln, umfangreich ist und häufigen Änderungen unterliegt, sind alle vier Ansätze für ein umfassendes Verständnis institutionellen Wandels notwendig. Die quantitative Messung des Reformumfangs, sowohl global im Sinne des Makro-Ansatzes als auch aufgeteilt nach Regelungsinhalt in Meso I, ist unter diesen Bedingungen notwendig für eine valide Beschreibung der Reformintensität sowie zur Identifikation von Reformen, die eine genauere Analyse erfordern. Die Unterscheidung von Reformtypen anhand ihrer Folgen für politische Prozesse und Ergebnisse im Sinne des Meso II-Ansatzes ist immer dann erforderlich, wenn Reformen verschiedenen Kausalmechanismen unterliegen und daher verschiedene Erklärungsmodelle erfordern, z. B. weil sich die betroffenen Akteure und ihre Motive unterscheiden. Der starke Detailfokus des Mikroansatzes schließlich erlaubt eine direkte Validierung theoretisch unterstellter Kausalmechanismen und erfasst institutionelle Details, die für die substanziellen Effekte institutioneller Regeln häufig von entscheidender Bedeutung sind.

Da allerdings nicht alle Studien institutionellen Wandel derart umfassend untersuchen wollen, diskutieren wir abschließend zwei begrenztere Strategien für Analysen, die vorrangig Detailerklärungen bzw. allgemeine Erklärungen anstreben. Untersuchungen, die eine möglichst detailgetreuen Beschreibung und Erklärung einzelner Reformen anstreben, also der „Logik der Tiefe“ (Gschwend und Schimmelfennig 
2007) verpflichtet sind, werden vorrangig den Mikroansatz verfolgen. Wenn die in die Analyse einbezogenen Regeln eng definiert sind (z. B. nur Agendasetzungsregeln im Plenum), kann auf eine quantitative Beschreibung von Veränderungen (Makro und Meso I) verzichtet werden. Sind die analysierten Regeln zudem so homogen, dass theoretisch ein einheitlicher Kausalmechanismus hinter allen Reformen angenommen werden kann, ist auch die Unterscheidung von Reformtypen im Sinne des Meso II-Ansatzes unnötig.

Die zweite Forschungsstrategie zielt auf allgemeine Erklärungen ab und ist im Sinne der „Logik der Breite“ (Gschwend und Schimmelfennig 2007) bereit, zugunsten eines größeren Anwendungsbereichs der Erklärung Detailunterschiede zu ignorieren. In diesem Fall sollte hauptsächlich auf die beiden Ansätze der Mesoebene zurückgegriffen werden, die relativ abstrakte Indikatoren zu Subgruppen von Regeln zur Verfügung stellen (beispielsweise die oben vorgestellten Reformen von Minderheitsrechten in Parlamenten, alle Reformen von Wahlsystemregeln zur Mandatsverteilung oder alle Veränderungen in Subsystemen des Wohlfahrtsstaats wie der Krankenversicherung). Der Makroansatz ist für Erklärungen in der Regel nicht geeignet, weil Institutionen in ihrem Design und ihren Folgen zu komplex sind, als dass sich substanziell relevante Fragen allein mit Makroindikatoren über einen großen und intern undifferenzierten Regelungskorpus beantworten ließen. Der Mikroansatz wiederum analysiert Details, die gemäß der „Logik der Breite“ als zu kleinteilig betrachtet und daher nicht modelliert und empirisch untersucht werden. Im Verlauf des Forschungsprozesses kann natürlich immer ein Aufsteigen in Richtung des Makro-Ansatzes zur Kontextualisierung oder ein Absteigen in Richtung des Mikro-Ansatzes zur Fundierung theoretisierter Zusammenhänge geboten erscheinen.

Die in diesem Artikel vorgestellten und für interessierte Forscher frei zugänglichen Werkzeuge (s. Fußnote 2) sind unabhängig vom Gegenstandsbereich auf alle Reformen formaler Institutionen anwendbar. ${ }^{12}$ Formale Regeländerungen schlagen sich stets als Änderungen von Texten nieder, die mit Hilfe unserer Textvergleichsund Verknüpfungsverfahren identifiziert werden können. Unsere automatisierten Verfahren führen zu großen Effizienzgewinnen im Prozess der Verknüpfung und sind weit weniger fehleranfällig als ein manuelles Vorgehen. Die Verknüpfungsinformation erleichtert zudem die weitere Kodierung und Analyse von Textänderungen. Gerade für große Textmengen, wie sie bei ländervergleichenden Analysen über lange Zeiträume hinweg und/oder bei der Untersuchung umfangreicher Regelungswerke entstehen, ist eine derartige (Teil-) Automatisierung des Forschungsprozesses dringend erforderlich. Die hier vorgestellten Werkzeuge haben sich in der Forschungspraxis bewährt und können weit über unsere Anwendung auf parlamentarische Regeln hinaus die systematische Analyse von Reformen großer Textkorpora voranbringen.

\footnotetext{
${ }^{12}$ Unsere Verfahren sind zudem nicht an einen bestimmten theoretischen Erklärungsansatz gebunden. Vielmehr dienen sie der Identifizierung von Reformen als abhängiger Variable, die anschließend je nach Fragestellung und theoretischem Interesse beispielsweise mit verschiedenen Spielarten des Neoinstitutionalismus (rational choice; historisch; soziologisch) erklärt werden können.
} 


\section{Literatur}

Allan, James P., und Lyle A. Scruggs. 2004. Political Partisanship and Welfare State Reform in Advanced Industrial Societies. American Journal of Political Science 48:496-512.

Benoit, Kenneth. 2007. Electoral Laws as Political Consequences. Explaining the Origins and Change of Electoral Institutions. Annual Review of Political Science 10:363-390.

Benz, Arthur, und Nathalie Behnke, Hrsg. 2009. Federalism and Constitutional Change. Special Issue. Publius 39 (2).

Binder, Sarah A. 1996. The Partisan Basis of Procedural Choice: Allocating Parliamentary Rights in the House, 1789-1990. American Political Science Review 90:8-20.

Blanchard, Philippe, Felix Bühlmann, und Jacques-Antoine Gauthier, Hrsg. 2014. Advances in Sequence Analysis. New York, NY: Springer.

Brouard, Sylvain. 2011. France: Systematic Institutional Advantage of Government in Law-Making. In The Role of Governments in Legislative Agenda Setting, Hrsg. Bjorn Erik Rasch, und George Tsebelis, 38-52. London: Routledge.

Christen, Peter. 2012. Data Matching. Heidelberg: Springer.

Cox, Gary W., und Mathew D. McCubbins. 2011. Managing Plenary Time: The U.S. Congress in Comparative Perspective. In The Oxford Handbook of the American Congress, Hrsg. George C. Edwards, Frances E. Lee, und Eric Schickler, 451-472. Oxford: Oxford University Press.

Diermeier, Daniel, und Keith Krehbiel. 2003. Institutionalism as a Methodology. Journal of Theoretical Politics 15:123-144.

Döring, Herbert. 1995. Time as a Scarce Resource: Government Control of the Agenda. In Parliaments and Majority Rule in Western Europe, Hrsg. Herbert Döring, 223-246. Frankfurt: Campus.

Fischer, Heinz. 1975. Die Reform der Geschäftsordnung des österreichischen Nationalrates nach dem Ende der großen Koalition. Zeitschrift für Parlamentsfragen 6:297-309.

Fischer, Heinz. 2002. Die Entwicklung der Geschäftsordnung des österreichischen Nationalrats seit 1996. In Der Rechtsstaat vor neuen Herausforderungen, Hrsg. Bernd-Christian Funk, 97-109. Wien: Verlag Österreich.

Gschwend, Thomas, und Frank Schimmelfennig. 2007. Lehren für den Dialog zwischen Theorie und Daten. In Forschungsdesign in der Politikwissenschaft, Hrsg. Thomas Gschwend, und Frank Schimmelfennig, 323-336. Frankfurt: Campus.

Harfst, Philipp. 2013. Changing the Rules of the Game: Determinants of Successful Electoral System Change in Central and Eastern Europe. International Political Science Review 34:427-443.

Helmke, Gretchen, und Steven Levitsky. 2004. Informal Institutions and Comparative Politics. A Research Agenda. Perspectives on Politics 2:725-740.

Huber, John D., und Charles R. Shipan. 2002. Deliberate Discretion? Cambridge: Cambridge University Press.

Kaiser, André. 2002. Mehrheitsdemokratie und Institutionenreform. Frankfurt: a. M.: Campus.

Katz, Richard S. 2005. Why Are There So Many (or So Few) Electoral Reforms? In The Politics of Electoral Systems, Hrsg. Michael Gallagher, und Paul Mitchell, 57-76. Oxford: Oxford University Press.

Korpi, Walter, und Joakim Palme. 1998. The Paradox of Redistribution and Strategies of Equality. Welfare State Institutions, Inequality, and Poverty in Western Countries. American Sociological Review 63:661-687.

Levenshtein, Vladimir I. 1966. Binary Codes Capable of Correcting Deletions, Insertions, and Reversals. Soviet Physics Doklady 10:707-710.

List, Johann-Mattis. 2014. Sequence Comparison in Historical Linguistics. Düsseldorf: Düsseldorf University Press.

Lutz, Donald S. 1994. Toward a Theory of Constitutional Amendment. American Political Science Review $88: 355-370$

Marschall, Stefan. 1999. Parlamentsreform. Opladen: Leske + Budrich.

Marschall, Stefan. 2004. Beziehungsspiele zwischen Parlament und Regierung - "Rules of the Game" und ihre Reform. In Kampf der Gewalten? Hrsg. Everhard Holtmann und Werner J. Patzelt, 313-332. Wiesbaden: VS Verlag für Sozialwissenschaften.

Mellado Prado, Pilar. 1983. El Nuevo Reglamento del Congreso de los Diputados. Revista de Derecho Político 18-19:225-244.

Müller, Wolfgang C. 2002. Parties and the Institutional Framework. In Political Parties in the New Europe, Hrsg. Kurt Richard Luther, und Ferdinand Müller-Rommel, 249-292. Oxford: Oxford University Press. 
Müller, Wolfgang C., und Ulrich Sieberer. 2014. Procedure and Rules in Legislatures. In The Oxford Handbook of Legislative Studies, Hrsg. Shane Martin, Thomas Saalfeld und Kaare W. Strøm, 311331. Oxford: Oxford University Press.

Ostrom, Elinor. 2005. Understanding Institutional Diversity. Princeton, NJ: Princeton University Press.

Pierson, Paul. 2004. Politics in Time. Princeton, NJ: Princeton University Press.

Renwick, Alan. 2011. Electoral Reform in Europe since 1945. West European Politics 34:456-477.

Schickler, Eric. 2000. Institutional Change in the House of Representatives, 1867-1998: A Test of Partisan and Ideological Power Balance Models. American Political Science Review 94:269-288.

Sieberer, Ulrich. 2006. Agenda Setting in the German Bundestag. A Weak Government in a Consensus Democracy. German Politics 15:49-72.

Sieberer, Ulrich und Wolfgang C. Müller. 2014. A Theory of Institutional Change in Parliaments: Understanding Institutional Reforms in Parliamentary Democracies in a Nested Games Framework. Manuskript, Konstanz/Wien

Sieberer, Ulrich, Wolfgang C. Müller und Maiko I. Heller. 2011. Reforming the Rules of the Parliamentary Game: Measuring and Explaining Changes in Parliamentary Rules in Austria, Germany, and Switzerland, 1945-2010. West European Politics 34:948-975.

Sieberer, Ulrich, Peter Meißner, Julia F. Keh und Wolfgang C. Müller. 2014a. Parliamentary Rule Changes in Europe: A Research Program. Manuskript, Konstanz/Wien.

Sieberer, Ulrich, Julia F. Keh, Peter Meißner und Wolfgang C. Müller. 2014b. Explaining Institutional Reforms of Minority Rights in Ten Western European Parliaments, 1945-2010. Paper presented at the 2014 APSA Annual Meeting, Washington, D.C., August 28-31, 2014.

Slapin, Jonathan B., und Sven-Oliver Proksch. 2014. Words as Data: Content Analysis in Legislative Studies. In The Oxford Handbook of Legislative Studies, Hrsg. Shane Martin, Thomas Saalfeld und Kaare W. Strøm, 126-144. Oxford: Oxford University Press.

Taagepera, Rein, und Matthew Soberg Shugart. 1989. Seats and Votes. New Haven, CT: Yale University Press.

Tsebelis, George. 1990. Nested Games. Berkeley, CA: University of California Press.

van der Loo, Mark P.J. 2014. The stringdist Package for Approximate String Matching. The R Journal 6: 111-122. 